condition $2 k \equiv 0(\bmod q-1)$ implies that $\left.2^{2 k}-2 \equiv-1(\bmod q).\right)$ Thus we have proved:

Lemma 2. The number $2(2 k-1) ! s_{k}$, when expressed as a fraction in lowest terms, has denominator $b_{k} k_{1}$.

Proof OF ThEOREM 2. If $r=4 k$ is a multiple of $2(q-1) q^{i}$, it follows that $q$ is of rank $k$. Hence $q$ divides $b_{k}$ and $q^{i}$ divides $k_{1}$; so that $q^{i+1}$ divides the denominator of $b_{k} k_{1}$. Together with Theorem 1, Corollary 2 this completes the proof.

\title{
THE CARTESIAN PRODUCT OF A CERTAIN NONMANIFOLD AND A LINE IS $E^{4}$
}

BY R. H. BING ${ }^{1}$

Communicated February 25, 1958

An upper semicontinuous decomposition $G$ of $E^{3}$ into points and tame arcs is defined in [1] such that the decomposition space $B$ is topologically different from $E^{3}$. Interesting properties of this space have also been given by Fort [4], Curtis $[2 ; 3]$, and Wilder [3]. We show that the cartesian product of the space $B$ and a line $E^{1}$ is topologically $E^{4}$. Perhaps the argument used is related to that employed by Arnold Shapiro to show that the cartesian product of a manifold described by Whitehead in [5] and a line is topologically $E^{4}$.

The arcs of the decomposition $G$ are intersections of double tori as shown in the figure. The solid double torus contains four double tori $T_{1}, T_{2}, T_{3}, T_{4}$ as shown; each $T_{i}$ in turn contains four double tori $T_{i 1}, T_{i 2}, T_{i 3}, T_{i 4}$ (not shown) imbedded in $T_{i}$ as $T_{1}, T_{2}, T_{3}, T_{4}$ were imbedded in $T$; more double tori are imbedded in the $T_{i j}$ 's; etc. The tame arcs of the decomposition $G$ are the components of

$$
T \cdot \Sigma T_{i} \cdot \Sigma T_{i j} \cdot \Sigma T_{i j k} \cdot \ldots \text {. }
$$

Although these tame arcs are mutually exclusive, it is not possible to get a 2 -sphere in $E^{3}$ that misses their sum and separates two of them. No topological cube in $T$ contains $T_{1}+T_{2}+T_{3}+T_{4}$.

When the cartesian product is taken, the extra dimension enables one to unravel certain linking handles in the sense that if $[a, b]$ is

${ }^{1}$ Work supported by the National Science Foundation under NSF Grant G3248 at the Institute for Advanced Study, Princeton, New Jersey. 


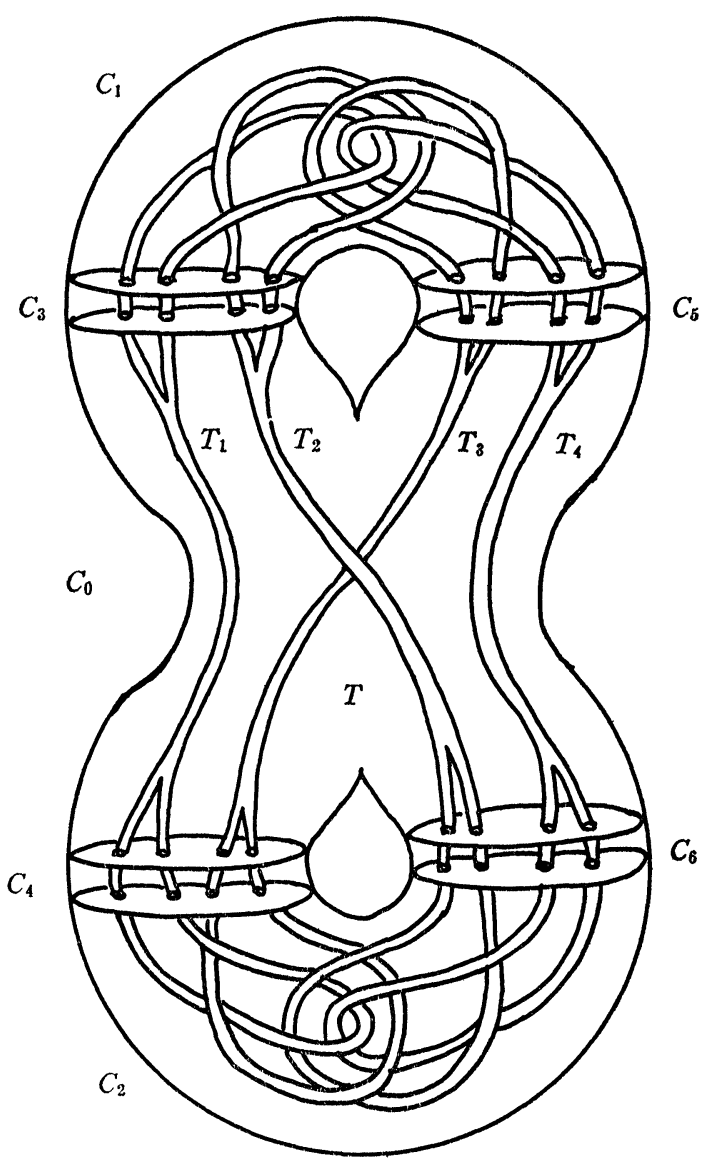

an interval and $\epsilon>0$, then there is a topological 4-cell in

$$
T \times[a-\epsilon, b+\epsilon]
$$

that contains $\left(T_{1}+T_{2}+T_{3}+T_{4}\right) \times[a, b]$.

In order to describe this topological 4-cell, we use eight disks to chop $T$ into seven topological cubes $C_{0}, C_{1}, C_{2}, C_{3}, C_{4}, C_{5}, C_{6}$ as shown in the figure. The topological 4-cell in $T \times[a-\epsilon, b+\epsilon]$ that contains $\left(T_{1}+T_{2}+T_{3}+T_{4}\right) \times[a, b]$ is the sum of the following thirteen 4-cells:

$$
\begin{array}{ll}
C_{0} \times[a-\epsilon, b+\epsilon], & \\
\left(C_{1}+C_{3}\right) \times[a-\epsilon, a-\epsilon / 2], & \left(C_{2}+C_{4}\right) \times[a-\epsilon, a-\epsilon / 2], \\
\left(C_{1}+C_{5}\right) \times[b+\epsilon / 2, b+\epsilon], & \left(C_{2}+C_{6}\right) \times[b+\epsilon / 2, b+\epsilon], \\
\left(T_{1} \cdot\left(C_{1}+C_{3}\right)\right) \times[a-\epsilon / 2, b], & \left(T_{2} \cdot\left(C_{1}+C_{3}\right)\right) \times[a-\epsilon / 2, b],
\end{array}
$$




$$
\begin{array}{ll}
\left(T_{3} \cdot\left(C_{1}+C_{5}\right)\right) \times[a, b+\epsilon / 2], & \left(T_{4} \cdot\left(C_{1}+C_{5}\right)\right) \times[a, b+\epsilon / 2], \\
\left(T_{1} \cdot\left(C_{2}+C_{4}\right)\right) \times[a-\epsilon / 2, b], & \left(T_{3} \cdot\left(C_{2}+C_{4}\right)\right) \times[a-\epsilon / 2, b], \\
\left(T_{2} \cdot\left(C_{2}+C_{6}\right)\right) \times[a, b+\epsilon / 2], & \left(T_{4} \cdot\left(C_{2}+C_{6}\right)\right) \times[a, b+\epsilon / 2] .
\end{array}
$$

The first of these thirteen 4-cells forms the main body of the sum, while the next four serve as wings onto which are fastened the last eight.

The fact that the cartesian products of the sum of double tori and intervals lie in such 4-cells enables one to describe in isotopy on $E^{3} \times E^{1}=E^{4}$ that shrinks sets of the sort $A \times w$ to small size where $A$ is a tame arc of $G$ in $E^{3}$ and $w \in E^{1}$. This shrinking is continued to a pseudo isotopy that shows that $B \times E^{1}$ is topologically $E^{4}$. Hence we have the following result in which the equality implies topological equivalence.

TheOREM. $B \times E^{1}=E^{4}$.

The noncompactness of $B$ is not a critical issue for if we let $\bar{B}$ denote the one point compactification of $B$ we have the following results.

Theorem. $\bar{B} \times E^{1}=S^{3} \times E^{1}$.

TheOREM. $\bar{B} \times S^{1}=S^{3} \times S^{1}$.

ThEOREM. The sum of two cones over the common base $\bar{B}$ is $S^{4}$.

The preceding results show that there are homeomorphisms of period 2 of $E^{4}$ and $S^{4}$ onto themselves whose fixed point sets are $B$ and $\bar{B}$ respectively.

\section{REFERENCES}

1. R. H. Bing, $A$ decomposition of $E^{3}$ into points and tame arcs such that the decomposition space is topologically different from $E^{8}$, Ann. of Math. vol. 65 (1957) pp. $484-500$.

2. M. L. Curtis, $A n$ imbedding theorem, Duke Math. J. vol. 24 (1957) pp. 349-352.

3. M. L. Curtis and R. L. Wilder, The existence of certain types of manifolds, to appear.

4. M. K. Fort, Jr., A note concerning a decomposition space defined by Bing, Ann. of Math. vol. 65 (1957) pp. 501-504.

5. J. H. C. Whitehead, A certain open manifold whose group is unity, Quart. J. Math. vol. 6 (1935) pp. 268-279.

UNIVERSITY OF WISCONSIN AND

INSTITUTE FOR ADVANCED STUdy 